\title{
Ephemeral-habitat colonization and neotropical species richness of Caenorhabditis nematodes
}

\author{
Céline Ferrari ${ }^{1}$, Romain Salle ${ }^{1}$, Nicolas Callemeyn-Torre ${ }^{1}$, Richard Jovelin ${ }^{2}$, Asher D. Cutter ${ }^{2^{*}}$ (1) \\ and Christian Braendle ${ }^{1^{*}}$
}

\begin{abstract}
Background: The drivers of species co-existence in local communities are especially enigmatic for assemblages of morphologically cryptic species. Here we characterize the colonization dynamics and abundance of nine species of Caenorhabditis nematodes in neotropical French Guiana, the most speciose known assemblage of this genus, with resource use overlap and notoriously similar external morphology despite deep genomic divergence.
\end{abstract}

Methods: To characterize the dynamics and specificity of colonization and exploitation of ephemeral resource patches, we conducted manipulative field experiments and the largest sampling effort to date for Caenorhabditis outside of Europe. This effort provides the first in-depth quantitative analysis of substrate specificity for Caenorhabditis in natural, unperturbed habitats.

Results: We amassed a total of 626 strain isolates from nine species of Caenorhabditis among 2865 substrate samples. With the two new species described here (C. astrocarya and C. dolens), we estimate that our sampling procedures will discover few additional species of these microbivorous animals in this tropical rainforest system. We demonstrate experimentally that the two most prevalent species (C. nouraguensis and C. tropicalis) rapidly colonize fresh resource patches, whereas at least one rarer species shows specialist micro-habitat fidelity.

Conclusion: Despite the potential to colonize rapidly, these ephemeral patchy resources of rotting fruits and flowers are likely to often remain uncolonized by Caenorhabditis prior to their complete decay, implying dispersal-limited resource exploitation. We hypothesize that a combination of rapid colonization, high ephemerality of resource patches, and species heterogeneity in degree of specialization on micro-habitats and life histories enables a dynamic co-existence of so many morphologically cryptic species of Caenorhabditis.

Keywords: Caenorhabditis astrocarya, Caenorhabditis dolens, Caenorhabditis elegans, Habitat sharing, Morphological crypsis, Metapopulations, Species co-existence, Species richness

\section{Background}

A long-standing question in ecology is how a diversity of species can coexist simultaneously in sympatry. One possible contributor to regional species co-existence is resource patch turnover, especially when species differ in their colonization and competitive abilities [1-3].

\footnotetext{
*Correspondence: asher.cutter@utoronto.ca; braendle@unice.fr

${ }^{1}$ CNRS, IBV, Inserm, Université Côte d'Azur, Nice, France

2 Department of Ecology and Evolutionary Biology, University of Toronto,

Toronto, ON M5S 3B2, Canada
}

With high patch turnover, the role of 'priority effects' in the order of species colonization plays a critical role in determining whether or not multiple species can coexist within a patch and across the landscape. How these factors come together is especially intriguing for speciesrich groups with similar ecological roles and strongly overlapping resource use. Therefore, it becomes crucial to characterize the species community composition and resource colonization capabilities of those species in natural systems. 
A hotspot of Caenorhabditis nematode diversity in the tropical rainforest of French Guiana, in particular, provides a compelling example in which many species occupy the same micro-habitats [4]. This speciose genus (> 50 species, K. Kiontke pers. comm.) exhibits frequent morphological crypsis and resource overlap among species, despite extreme genomic divergence between one another [5-8]. Caenorhabditis nematodes thus provide a tantalizing ecological model for metapopulation dynamics as a contributor to species co-existence, in addition to their role as important biomedical model organisms [9]. All species of Caenorhabditis are microbivorous, found most commonly within decaying vegetal matter. These ephemeral resource patches should underlie a highly dynamic metapopulation structure for Caenorhabditis on the landscape, especially in tropical environments with rapid decay rates [10-13]. However, among the many poorly understood features of their natural history, the details of patch colonization, occurrence with patch age, and patch substrate specificity form especially crucial factors for understanding the abundance and diversity of these organisms.

Natural Caenorhabditis populations have become increasingly studied during the past few years, with a primary focus on C. elegans [5, 12-18]. These efforts have led to the discovery of many new species and the establishment of hundreds of cryopreserved Caenorhabditis wild isolates available for study from across the world $[6,10,11,19]$. In turn, these live specimen collections have prompted studies of population genomics [20,21], genome-wide association mapping of biologically important traits [22], discovery of and experimentation with natural pathogens $[11,23]$, and the seasonal turnover of species [16]. Together they provide the unique opportunity to integrate the extensive knowledge of Caenorhabditis cellular development, neurobiology, and molecular genetics with ecological characteristics and evolutionary processes.

In-depth analyses of natural Caenorhabditis populations, however, have been limited to mainland France and Germany, with a focus on the three predominant species of this region: C. elegans, C. briggsae and C. remanei $[10-12,15-17,24-26]$. The vast majority of these surveys were collected in anthropogenic habitats, such as orchards or garden compost heaps and little information is available for unimpacted habitats. However, the analysis of habitats unperturbed by extensive human activity is crucial to understand the diversity and abundance of these organisms. Documentation of natural populations in tropical regions is less secure, despite providing epicenters of biodiversity from the levels of genetic variability to species richness [4, 10,27]. Previous study of Caenorhabditis from tropical French Guiana brought more general ecological questions to the fore [4, 27]. After identifying the most locally-common species, this work demonstrated co-occurrence of distinct species and genotypes within individual micro-habitat patches, and quantified DNA sequence variability for the two self-fertilizing species found there [4, 27]. Consequently, quantitative analysis of species richness and abundance, species-specificity of micro-habitat substrates, phoretic associations, and dispersal dynamics represent essential outstanding problems.

To characterize the dynamics and specificity of colonization and exploitation of ephemeral resource patches, we conducted manipulative field experiments and the largest sampling effort to date for Caenorhabditis outside of Europe [12, 15-17, 24-26]. By quantifying colonization rates and distinct species incidence among micro-habitats, we provide a foundational view of key factors in the metapopulation dynamics that we predict to be important in enabling local and regional species co-existence.

\section{Methods \\ Collection of samples and analysis}

We collected samples during field expeditions in April 2013, August 2014 and August 2015. Most samples were collected in primary rainforest of the Nouragues Natural Reserve $\left(4^{\circ} 5^{\prime} \mathrm{N}, 52^{\circ} 41^{\prime} \mathrm{W}\right)$, close to the two CNRS field stations "Inselberg" and "Parare," $8 \mathrm{~km}$ apart. In addition, we conducted opportunistic sampling of rotting fruit/ flower substrates and invertebrates from other sites in French Guiana, mostly in primary and secondary forests of coastal regions $(2013,2015)$ and in the surroundings of the village Saül in central French Guiana (2015) (Fig. 1). Additional file 1 lists all strain isolates collected, with corresponding details on sampling sites and substrate characteristics. All isolates were cryopreserved and are available upon request.

\section{Identification of species, isolate establishment, and species richness estimation}

For substrate sampling, nematode isolation, and species identification, we followed previously established protocols $[4,28,29]$. In brief, we stored samples in sealed plastic bags prior to analysis in the laboratory; a small subset of samples was directly isolated on site. Based on measurements of substrate samples from collections in 2013, they weighed $\sim 20 \mathrm{~g}$ on average and ranged from $\sim 5 \mathrm{~g}$ to $\sim 100 \mathrm{~g}$. To isolate nematodes, substrate samples were placed on Nematode Growth Medium (NGM) plates inoculated with the E. coli strain OP50 [30]. This procedure allows efficient isolation of a Caenorhabditis species from each sample, but is biased against identifying multiple species, in particular, slow-growing 


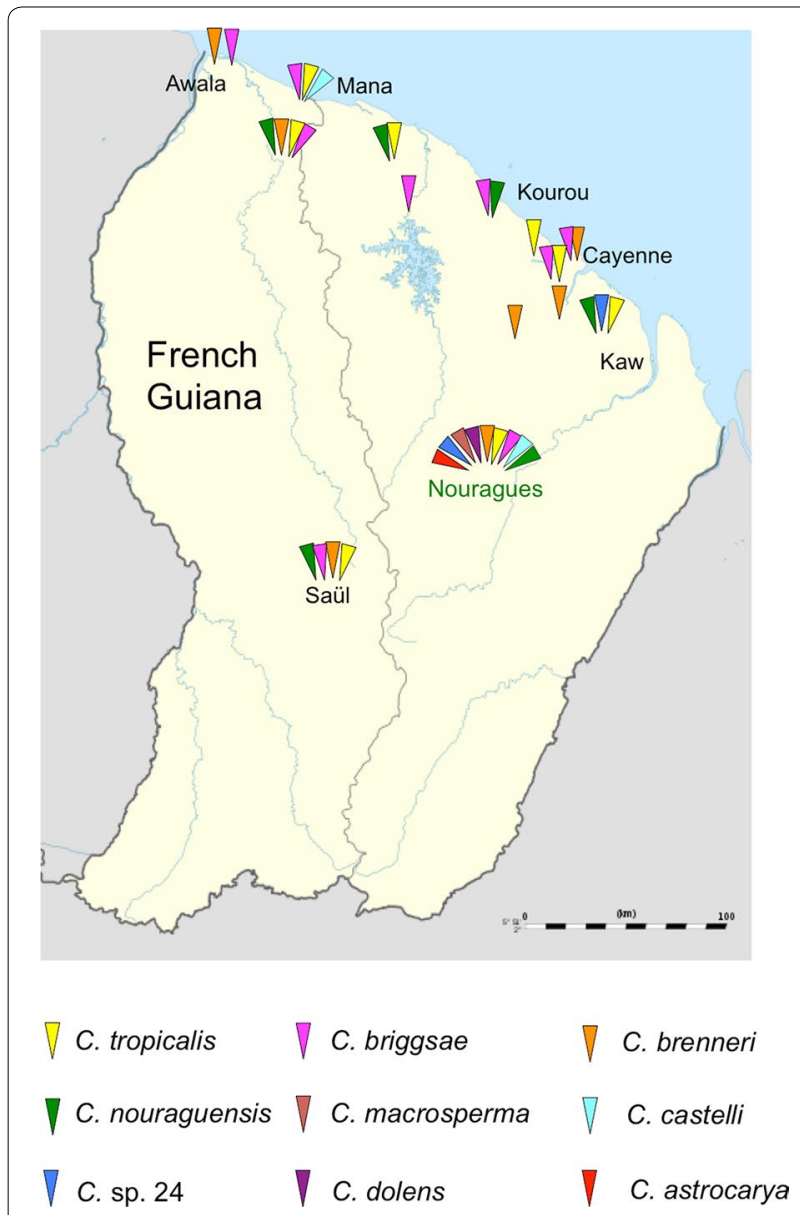

Fig. 1 Overview of Caenorhabditis species distribution in French Guiana (2009-2015), including data of [4]

species. Caenorhabditis genus identity was first established through microscopy analysis of morphology [31], and species identity was then confirmed using sequencing of the ITS2 region (Internally Transcribed Spacer) between the $5.8 \mathrm{~S}$ and $28 \mathrm{~S}$ rDNA genes, as previously described $[5,6]$. We used primers 5.8S-1 (5'-CTGCGTT ACTTACCACGAATTGCARAC) and KK28S-4 (5'-GC GGTATTTGCTACTACCAYYAMGATCTGC) that amplify a fragment of approximately $2 \mathrm{~kb}$, which was then partially sequenced using the forward PCR primer and the sequencing primer KK-28S-22 (5-CACTTTCAAG CAACCCGAC) $[6,31]$. The sequence of the ITS2 fragment provides a reliable species identification tag as it is highly divergent between, but not within, Caenorhabditis species [6]. Partial ITS2 sequences of the two new species are deposited in GenBank: MF940414 (C. dolens) and MF940415 (C. astrocarya).

All isolates were derived from a single hermaphrodite at the larval stage (for androdioecious species) or an adult, mated female (for gonochoristic species). All isolates were then cryopreserved (see Additional file 1 for complete list of isolates).

We estimated total species richness using the Chao2 estimator with EstimateS v. 9.1.0 [32].

\section{Identification and description of novel species}

We provide descriptions for two new species, which were provisionally termed $C$. sp. 37 and $C$. sp. 42 . We followed the current standard for new species description in this group based on molecular barcodes and biological species inference from mating tests [5]. ITS2 barcode sequences for both species are highly distinct from all currently known Caenorhabditis species, supporting their new species status. Nevertheless, following previously described protocols [6], we carried out reciprocal mating crosses with species that show strongest ITS2 sequence similarity to affirm reproductive isolation and identity as distinct biological species (see below).

\section{Nomenclatural acts}

The electronic edition of this article conforms to the requirements of the amended International Code of Zoological Nomenclature, and hence the new names contained herein are available under that Code from the electronic edition of this article. This published work and the nomenclatural acts it contains have been registered in ZooBank, the online registration system for the ICZN. The ZooBank LSIDs (Life Science Identifiers) can be resolved and the associated information viewed through any standard web browser by appending the LSID to the prefix "http://zoobank.org/". The LSID for this publication is urn:lsid:zoobank.org:pub:06DBDCA0-DD6043BA-9A99-14F0748D4FE2. The electronic edition of this work was published in a journal with an ISSN.

\section{Substrate incidence of Caenorhabditis}

To obtain quantitative estimates of Caenorhabditis presence on different types of substrates (native fruit versus litter), we sampled 56 spots across the trail network of the Inselberg station in the Nouragues Natural Reserve (2013) (Fig. 2, Additional file 2). At each spot, we defined a sampling area of approximately $20 \mathrm{~m}^{2}$ within which we collected $\sim 10$ litter samples and up to five fruit samples, the latter consisting of diverse, usually strongly decayed fruit material on the forest floor.

\section{Micro-habitat colonization and age-dependent incidence}

To assess Caenorhabditis colonization of bait samples (Additional file 3), we distributed pieces of surface-sterilized oranges (each piece 1/16th of a large orange) at 72 intervals spaced approximately $30 \mathrm{~m}$ apart along the trail network of the Parare station in the Nouragues Natural Reserve (2014). Oranges were chosen for logistical 

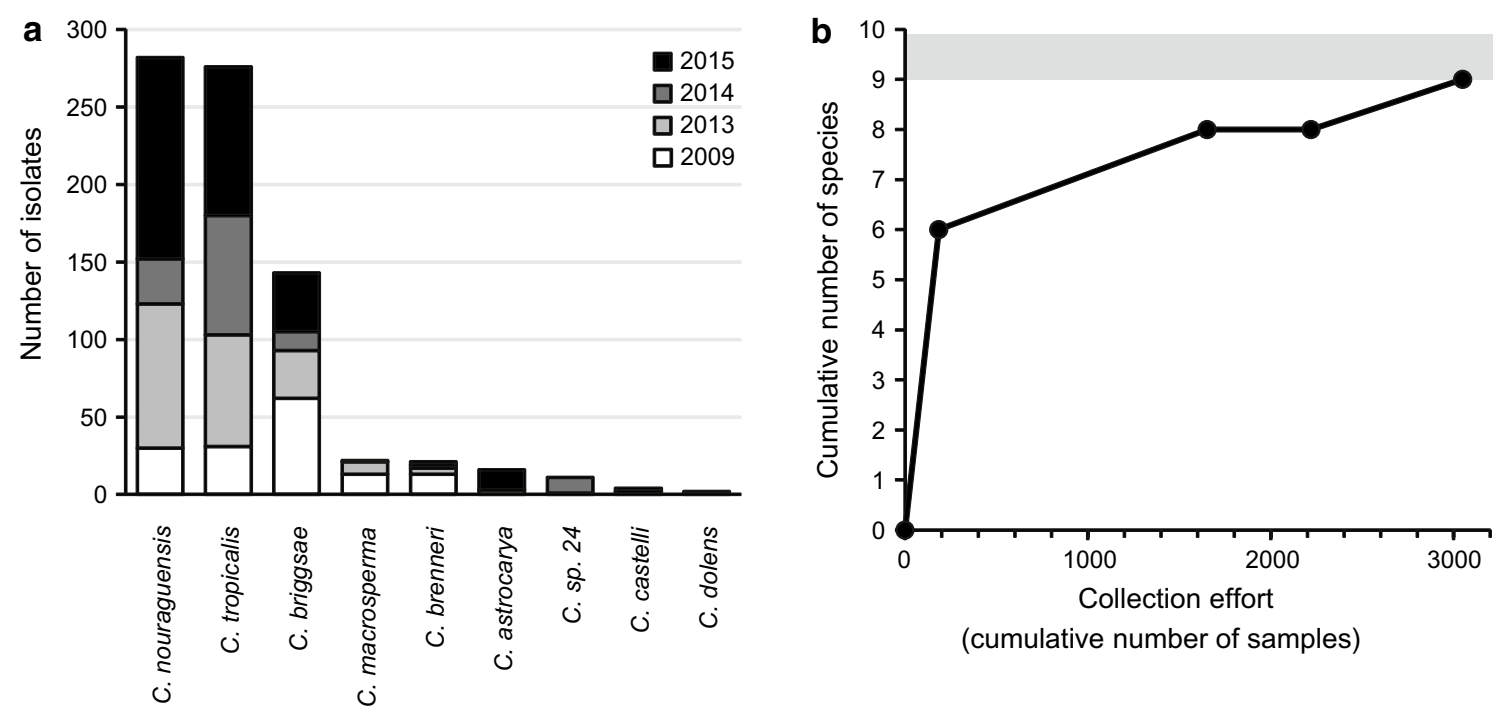

Fig. 2 Species diversity and abundance of Caenorhabditis nematodes collected in French Guiana. a Species rank-abundance distribution for Caenorhabditis isolates collected from 2009 to 2015. b Collectors curve of species identified in French Guiana as a function of sampling effort from 2009 to 2015. The confidence interval for the Chao2 estimate of species richness is indicated by the gray band

reasons as a standard readily-accessible fruit that could easily be sterilized and detected visually on the forest floor. At each spot, we distributed three groups of three orange pieces on the ground within an area of approximately $3 \mathrm{~m}^{2}$, with a single sample representing a group of three orange pieces, which were placed together into a single plastic bag 4 days after. Therefore, we collected three independent bait samples at each spot (total samples: $\mathrm{N}=216$ ).

To assess Caenorhabditis colonization rates of bait samples at localities that had abundant rotting fruit/ flower substrates on the ground (Additional file 4), we selected five localities near the Parare station and 18 localities near the Inselberg station (Nouragues Natural Reserve). At each locality, we distributed five bait samples (orange and banana pieces) within an area of approximately $5 \mathrm{~m}^{2}$. After 4 days, bait samples as well as five native fruit/flower samples were collected and stored in plastic bags for further analysis.

To examine Caenorhabditis colonization as a function of substrate age, we focused on a single locality where Clusia flowers (likely Clusia palmicida) at different stages of decay covered the ground (Inselberg station, Nouragues Natural Reserve). We haphazardly collected 70 flower samples across the entire $\sim 75 \mathrm{~m}^{2}$ area from each of three clearly distinct, progressive stages of decay ( $\mathrm{N}=210$; Fig. 4, Additional file 5). The freshest decay stage comprised fleshy petals and intact floral resin [33], with the latest stage being composed primarily of fibrous plant tissue. We then assessed species incidence on each flower sample. In addition, for a subset of these samples containing Caenorhabditis ( $\mathrm{N}=21)$, we immediately placed samples on NGM plates seeded with $E$. coli and determined number and stages of individuals $5-7 \mathrm{~h}$ later (Additional file 6).

\section{Results}

Through the combined effort of targeted sampling of native substrates, experimental baits, and opportunistic collection in the Nouragues Natural Reserve and throughout French Guiana across 3 years, we amassed a total of 626 strain isolates from nine species of Caenorhabditis among 2865 substrate samples (Figs. 1, 2a, Additional file 1). This represents the largest collection effort for Caenorhabditis to date outside of mainland Europe, and the single largest collection effort for nonanthropogenically modified habitats. Integrating the sampling and species discovery efforts in this study with a previous collection leads to a species richness upper 95\% confidence interval bound of 9.88 Caenorhabditis in French Guiana (Chao2 estimator; Fig. 2b). This result suggests that at most one additional species is likely to be discovered given current sampling procedures. Three species provide first records in French Guiana and two of these species are new to science (Caenorhabditis dolens n. sp. $=$ C. sp. 37 and Caenorhabditis astrocarya n. sp. $=C$. sp. 42, see below). Four species were most widespread by being present at numerous inland and coastal localities (C. nouraguensis, C. tropicalis, C. briggsae, and $C$. brenneri), with the gonochoristic $C$. nouraguensis and the androdioecious $C$. tropicalis being most abundant (Fig. 2a). Thus, French Guiana harbours a total of 
five endemic species to date (C. nouraguensis, C. macrosperma, $C$. castelli, $C$. dolens $\mathrm{n} . \mathrm{sp} .=C$. sp. 37 and $C$. astrocarya $\mathrm{n} . \mathrm{sp} .=C$. sp. 42 ) in addition to the presence of three cosmopolitan species (C. tropicalis, C. briggsae, C. brenneri) and one species found previously elsewhere in the New World tropics (C. sp. 24 found in Panama, M. Rockman, pers. comm.).

\section{Substrate incidence of Caenorhabditis}

We first aimed to test for quantitative differences in the prevalence of Caenorhabditis between decaying vegetal micro-habitats within a given site, namely between leaf litter and rotting fruit. Across 560 leaf litter samples from 56 locations covering about $4 \mathrm{~km}^{2}$ (Fig. 3), we isolated only 2 species of Caenorhabditis from just $2.7 \%$ of the samples (C. nouraguensis, C. tropicalis), with 43 of the 56 locations lacking Caenorhabditis entirely in litter samples (Additional file 2). By contrast, we isolated three Caenorhabditis species among $20.6 \%$ of 209 rotting fruit samples from 31 of the 56 locations $(C$. nouraguensis, $C$. tropicalis, C. briggsae) (Additional file 2). Just three of the locations contained Caenorhabditis in litter samples only, in contrast to 17 of the 56 locations that had Caenorhabditis found exclusively in fruit samples (Fig. 3). Overall, Caenorhabditis species were thus more frequently found in fruit samples (43 of 209 samples) than in litter samples (15 of 560 samples) (Fisher's Exact Test, $\mathrm{P}<0.0001$ ). These results provide quantitative support for the idea that Caenorhabditis occurs more frequently in highnutrient rotting micro-habitats $[10,11,14]$. At six of the 56 locations, we isolated $C$. nouraguensis and C. tropicalis from the same samples (Fig. 3). Non-Caenorhabditis nematodes (predominantly species of the Rhabditidae and Diplogasteridae) were found in most litter samples $(98 \%)$ and fruit samples (88\%) (Additional file 2), the latter consisting mostly of strongly decayed, rather than fresh fruit substrates.

\section{Micro-habitat colonization and substrate age-dependent incidence}

We next performed a series of field experiments to quantify colonization of fresh substrates by Caenorhabditis. Overall, we isolated Caenorhabditis on $9.7 \%$ of a total of 216 bait samples (C. tropicalis, C. briggsae, C. nouraguensis), with a range of 4 to $44 \%$ of baits having been colonized across the transects (Additional files 3 and 7). We calculate that $C$. nouraguensis and $C$. tropicalis colonized the baits as new resource patches at a rate of $~ 2.5-7.5 \%$ baits/day. We isolated two species (C. nouraguensis and C. tropicalis) from one bait sample, and two species $(C$. briggsae and C. tropicalis) from two samples at the same spot. However, this is an underestimate of the co-incidence of species because our isolation protocol is biased against finding multiple species per sample. Only a single bait sample was colonized by a non-Caenorhabditis nematode.

Having established that Caenorhabditis occurs readily on both native substrates of unknown age and on fresh baits, we next tested whether fresh fruit baits got more efficiently colonized by Caenorhabditis (C. tropicalis, C. briggsae, C. nouraguensis, C. sp. 24) when they were positioned near to locations on the rainforest floor with a high natural density of rotting fruit or flowers compared to random locations. Indeed, $30.1 \%$ of baits $(\mathrm{N}=123$ from a total of 23 spots) near to high densities of rotting native fruit or flowers contained Caenorhabditis after 5 days (Additional file 4), compared to just 9.7\% of randomly-located bait samples $(\mathrm{N}=216)$ (Fisher's Exact Test, $\mathrm{P}<0.0001$ ) (Additional file 3). Baits and native fruits in the same vicinity did not differ in the incidence of Caenorhabditis (30.1\% on baits versus $19.8 \%$ on native fruits) (Fisher's Exact Test, $\mathrm{P}=0.09$ ). In contrast, Non-Caenorhabditis nematodes were more common on the native fruit/flower substrates (57 of 116 baits colonized) than on the fresh baits (28 of 123 baits colonized) (Fisher's exact test, $\mathrm{P}<0.0001$; Additional file 4). These findings implicate existing rotting fruit/flower as an important dispersal source for Caenorhabditis in colonizing new micro-habitats.

As a final means of characterizing the incidence of $\mathrm{Cae}$ norhabditis as a function of substrate age, we quantified nematode species among 210 Clusia flowers in three distinct phases of decay (Fig. 4, Additional file 5). We found a striking effect of substrate decay stage on the presence of $C$. nouraguensis: more than half of the 70 freshest samples had been colonized (54\%), but just 13 and $4 \%$ of samples in increasingly more advanced states of decay contained this species (Fig. 4). C. tropicalis was rarer in these samples overall, but was never found on those Clusia samples in the most-advanced stage of decay (Fig. 4). By contrast, non-Caenorhabditis nematodes were least common on the freshest samples of Clusia (Fig. 4). Characterizing a subset of Clusia flowers containing Caenorhabditis $(\mathrm{N}=21)$ immediately on site, we found that most of these positive samples were colonized by starved adults rather than juvenile or dauer stages (Additional file 6). Only three of 21 samples contained more than 40 individuals of mixed age classes, indicative of proliferation, suggesting that colonization of fresh resource patches get founded by very few individuals. This low incidence of proliferating nematode populations contrasts with C. elegans on rotting apples in orchards [18]. 
All fruit and litter samples ( $\mathrm{N}=769)$

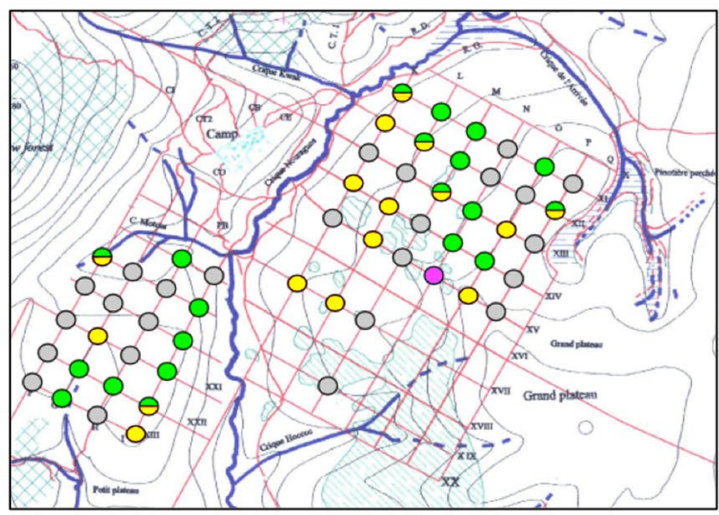

Fruit samples only ( $\mathrm{N}=209)$

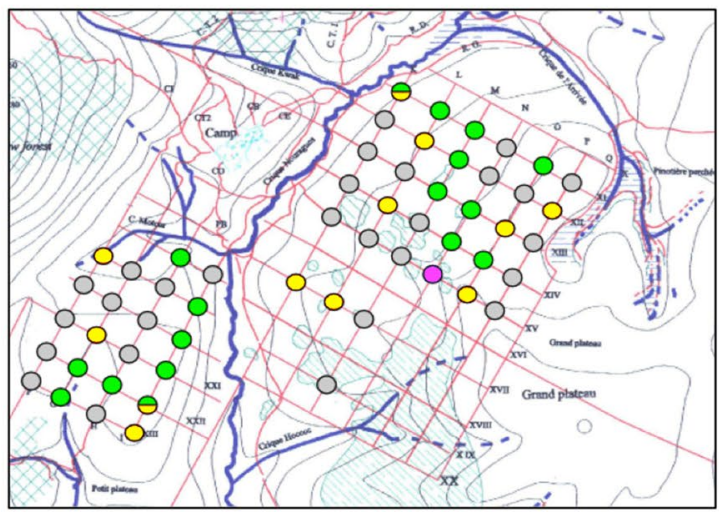

Intersections with Caenorhabditis exclusively found in fruit samples

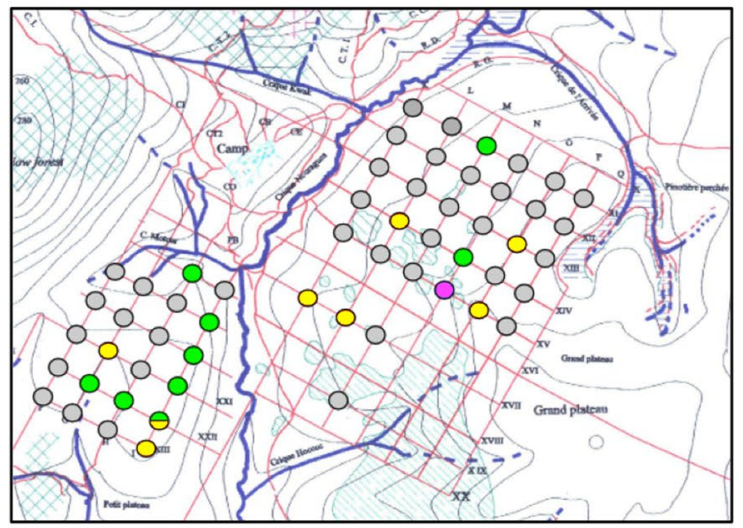

C. nouraguensis

$\bigcirc$ C. tropicalis

C. briggsae

$\bigcirc$ negative sample
Litter samples only ( $\mathrm{N}=560)$

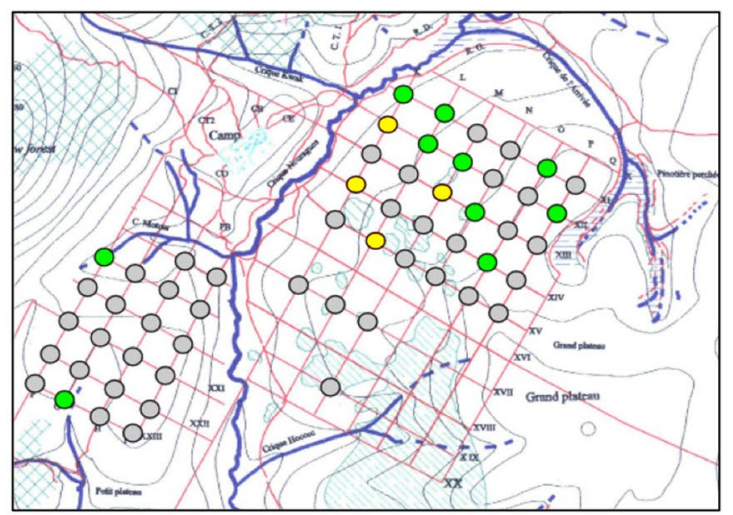

Intersections with Caenorhabditis exclusively found in litter samples

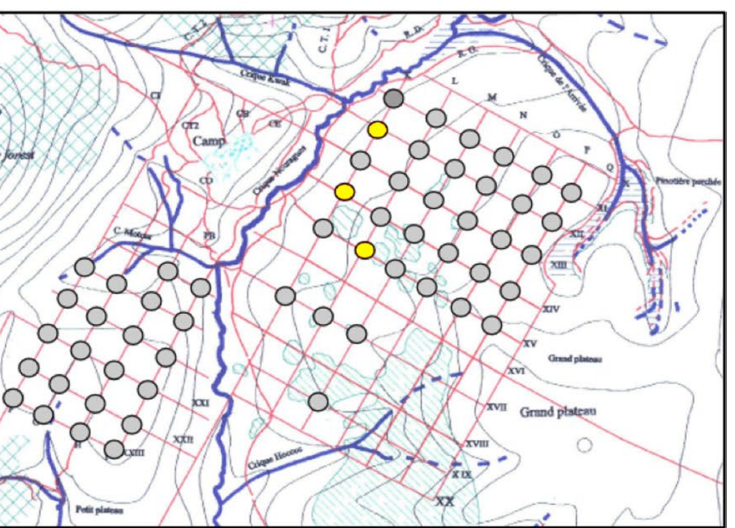

Fig. 3 Substrate incidence of Caenorhabditis (Inselberg site, Nouragues Natural Reserve). Quantitative differences in the incidence of Caenorhabditis in decaying leaf litter versus rotting fruit. Red grid lines in sampling area are spaced $\sim 100 \mathrm{~m}$ apart

Landscape-scale assessment of neotropical species richness for Caenorhabditis

We complemented targeted and experimental characterization of Caenorhabditis substrates with opportunistic sampling of diverse ephemeral-habitat substrates throughout the Nouragues Natural Reserve (Additional 

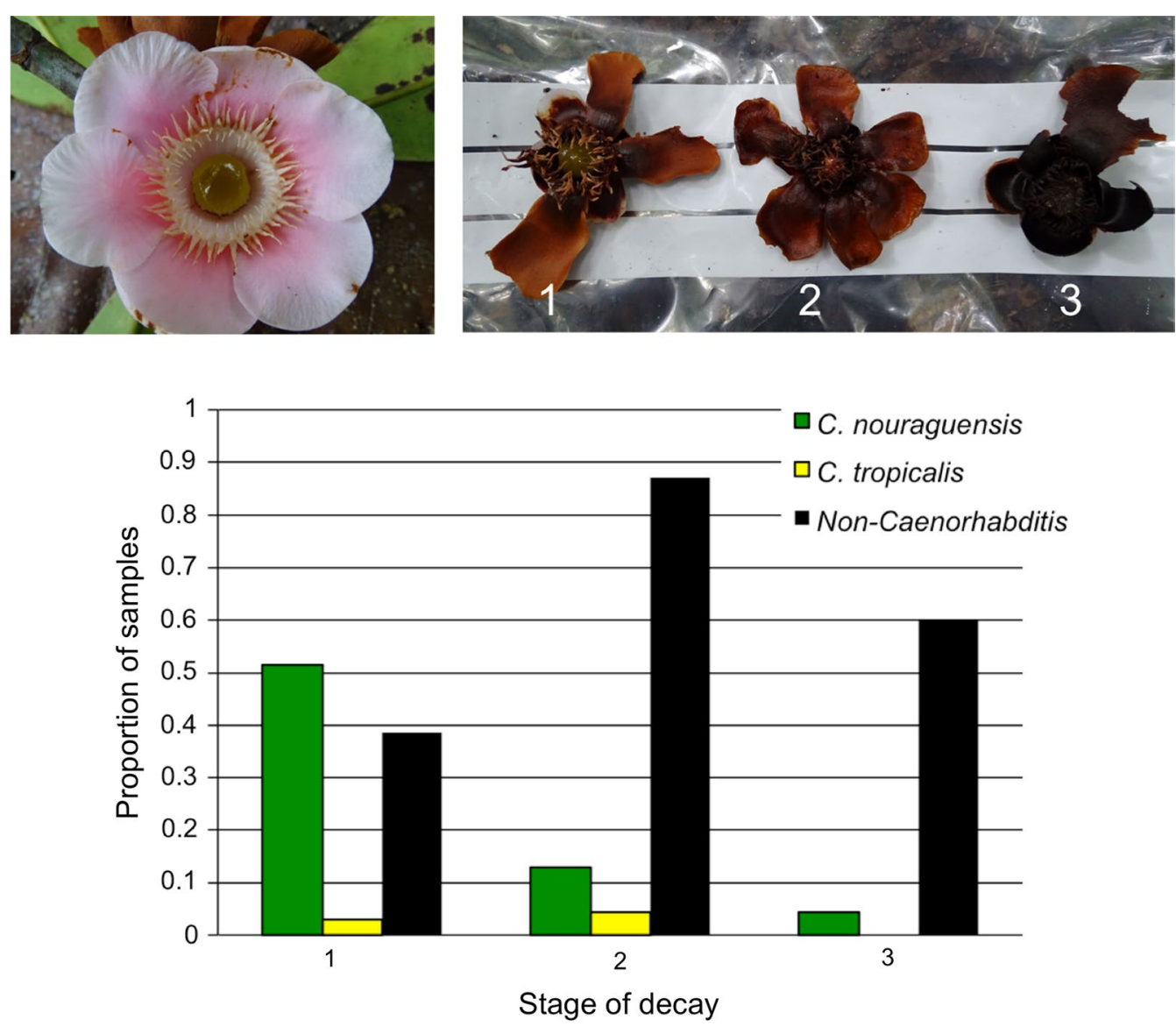

Fig. 4 Caenorhabditis colonization of Clusia flowers at three distinct stages of decay (1: slight decay, brown colour with intact floral resin, 2: intermediate decay, brown colour with no floral resin; 3: strong decay, black colour with no floral resin). Incidence of nematodes based on sampling 70 flowers from each decay stage. Decay stage differs significantly for presence of $C$. nouraguensis (Fisher's Exact Test $P<0.0001$ ) and non-Caenorhabiditis (Fisher's Exact Test $\mathrm{P}<0.0001$ ). Image on top-left shows a fresh Clusia flower

files 8, 9, 10) and French Guiana more broadly (Fig. 1; Additional file 11). The more diverse substrate collections in the Nouragues Natural Reserve yielded isolation of five rarer species from 690 samples, in addition to the four more common species found in our targeted substrate sampling (as detailed in the previous section) (Additional file 8). Outside of the Nouragues Natural Reserve, we recovered 151 isolates from six species of Caenorhabditis, deriving from 210 samples from a locality in inland Saül and 247 samples from multiple localities along the Atlantic coast of French Guiana (Additional file 11). These six species comprise a subset of those nine Caenorhabditis found within the Nouragues Natural Reserve (Fig. 1; Table 1), indicating that the species encountered throughout the regional scale can also be found from intensive sampling of a single locality.

All three of the most common species were isolated in every collection period $(2013,2014,2015)$, including a previous sampling effort in 2009 [4] (Fig. 2a). However, the species sampled most frequently differed across years (C. nouraguensis in 2013 and 2015, C. tropicalis in 2014, C. briggsae in 2009; Fig. 2a). We are unsure whether differences in the most abundant species reflects predictable seasonal differences across the sampling periods, stochastic changes, subtle differences in the cross-section of substrate samples collected, or longer-term trends in relative species rank abundance. Each of the remaining rarer species failed to be sampled in at least one collection episode, with the exception of C. brenneri, which was sampled in all collection periods in more humanassociated locations. The species rank-abundance distribution for Caenorhabditis in French Guiana follows the typical pattern for low evenness of a few common species and many more rare species (Fig. 2a) [34], though no other species rank-abundance curves are available for comparison with Caenorhabditis in other regions.

Our opportunistic sampling focused primarily on rotting flower and fruit substrates, yielding a total of 412 


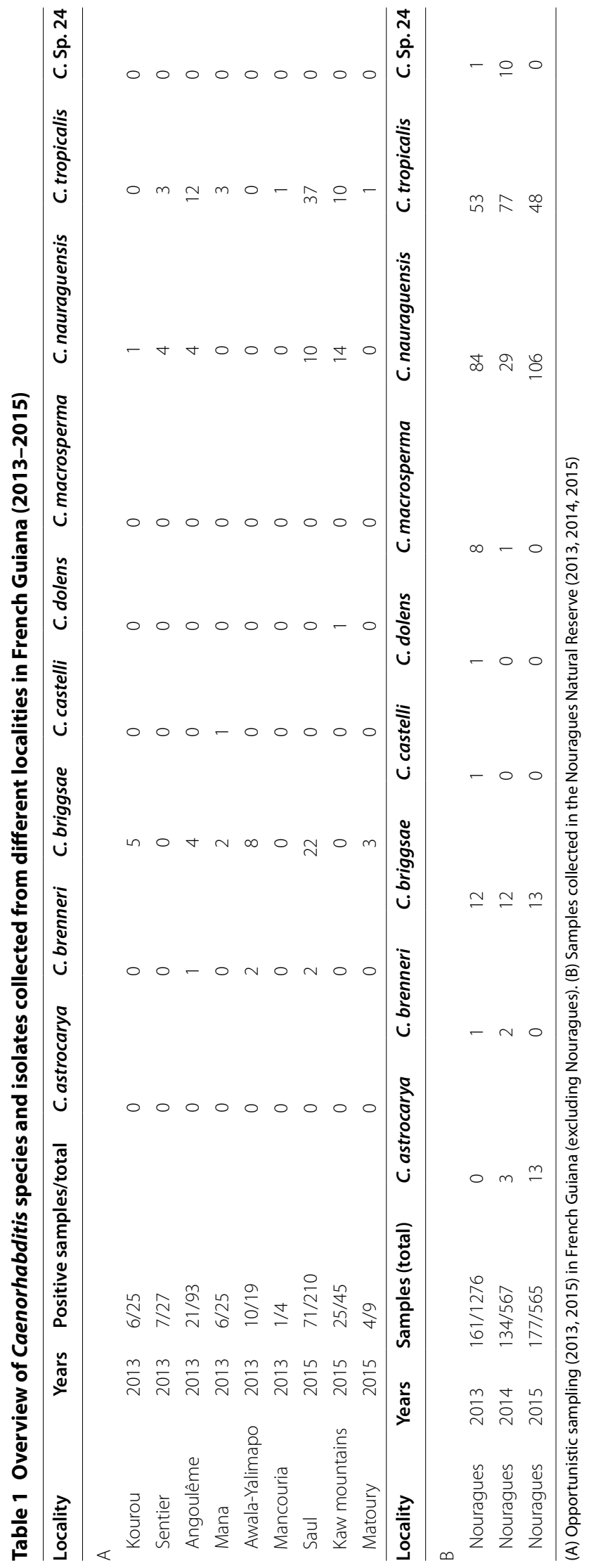


Caenorhabditis isolates. We did not detect obvious signs of species substrate specificity besides the association of the novel species $C$. astrocarya with Astrocaryum palms. The most commonly sampled substrates were Clusia spp. flowers from which we extracted 68 isolates composed of the dominant species, C. nouraguensis $(\mathrm{N}=33)$ and $C$. tropicalis $(\mathrm{N}=34)$ (plus one C. briggsae isolate) (Additional file 1). Similarly, C. nouraguensis and C. tropicalis were overall highly prevalent in both fruits and flowers. From collected plant stems containing Caenorhabditis $(\mathrm{N}=18$ ), however, we extracted 17 C. tropicalis (and one C. briggsae) isolates but never C. nouraguensis (Additional file 1). In addition, analysis of 58 invertebrate samples yielded seven Caenorhabditis isolates, including $C$. nouraguensis, C. macrosperma and C. tropicalis (12\% incidence, found on insects and millipedes; Additional files 8 and 11).

\section{Discovery and description of two new species of Caenorhabditis}

Our collection efforts yielded isolation of two species of Caenorhabditis previously unknown to science. Here we provide species descriptions of them, following new species description in this group based on molecular barcodes and biological species inference from mating crosses [5].

Caenorhabditis dolens Braendle \& Cutter sp. nov. urn:Isid:zoobank.org:act:4D39DD5D-2120-42E5-85210F35D8710592 = Caenorhabditis sp. 37.

The type isolate by present designation is NIC394. The species is delineated and diagnosed by the fertile cross with the type isolate NIC394 in both cross directions, yielding fertile hybrid females and males that are interfertile and cross-fertile with their parent strains. The species reproduces through males and females. This species differs by ITS2 DNA sequences from all other species in [6] and [5] (Genbank MF940414). Note that these DNA sequences may vary within the species. From molecular data, this species falls into the Drosophilae supergroup of Caenorhabditis with the closest known species being C. angaria, C. castelli and C. sp. 8 [3]. Reciprocal crossing experiments of $C$. dolens NIC394 with strains of these and other Caenorhabditis species did not result in any larval progeny (Additional file 12). Nomarski microscopy images of adult female and male morphological structures of C. dolens NIC394 are shown in Additional file 13. This species exhibits a spiral mating position, as observed in most closely related species [3]. The type isolate was collected in the Nouragues Natural Reserve (French Guiana) in 2013 from decaying fruit; no other isolates are currently known. The type culture specimens are deposited at the Caenorhabditis Genetics Center (https://cgc. umn.edu/).
Caenorhabditis astrocarya Braendle \& Cutter sp. nov. urn:lsid:zoobank.org:act:EBE4FF82-F906-4237-9290C84431F17D7D = Caenorhabditis sp. 42.

The type isolate by present designation is NIC1040. The species is delineated and diagnosed by the fertile cross with the type isolate NIC1040 in both cross directions, yielding highly fertile hybrid females and males that are interfertile and cross-fertile with their parent strains. The species reproduces through males and females. This species differs by ITS2 DNA sequences from all other species in [6] and [5] (Genbank MF940415). Note that these DNA sequences may vary within the species. From molecular data, this species falls into the Drosophilae supergroup of Caenorhabditis with the closest known species being C. guadeloupensis [3]. Reciprocal crossing experiments of C. astrocarya NIC1040 with strains of this and other Caenorhabditis species did not result in any larval progeny (Additional file 12). Nomarski microscopy images of adult female and male morphological structures of $C$. astrocarya NIC1040 are shown in Additional file 14. This species exhibits a parallel mating position. The type isolate was collected from the inflorescence of Astrocaryum palms (likely Astrocaryum paramaca) in the Nouragues Natural Reserve (French Guiana) in 2014 (Additional file 15). Additional isolates (Additional file 1) were collected in the same location, exclusively from fresh and rotten inflorescence stalks on Astrocaryum plants in 2014 and 2015. The palm inflorescences were frequently co-inhabited by abundant weevils (and may also harbour other Caenorhabditis species, such as C. tropicalis, $C$. nouraguensis or C. briggsae) (Additional file 1). The type culture specimens are deposited at the Caenorhabditis Genetics Center (https://cgc.umn.edu/).

\section{Discussion}

Deciphering the drivers of biodiversity and species coexistence first requires characterization of species richness and abundance, as well as detailed understanding of resource use and dispersal. To these ends for Caenorhabditis nematodes, a group most famous otherwise as biomedical model organisms, we conducted the largest biodiversity survey and field quantification of resource patch colonization in habitat unperturbed by human influence. Combining opportunistic sampling with quantitative, experimental methods, we collected nearly 3000 substrate samples and identified nine species of Caenorhabditis in French Guiana, two species of which are new to science (C. astrocarya $\mathrm{n}$. sp. and C. dolens $\mathrm{n}$. sp.; also, $C$. sp. 24 remains undescribed and was isolated previously in Panama, M. Rockman pers. comm.). These species are roughly evenly divided among the three major phylogenetic subgroups within the genus (2 Japonica group species, 3 Elegans group species, 4 Drosophilae 
supergroup species) [6, 10]. Given current sampling procedures, we estimate that we are unlikely to discover more than one new additional species from similar habitats in this locality, which represents the greatest biodiversity hotspot for Caenorhabditis described to date.

The three most common species overall were also the most common species in every sampling year, and include both endemic (C. nouraguensis), tropically widespread (C. tropicalis), and globally distributed species ( $C$. briggsae). Four of the rarer species are endemic to French Guiana given the current neotropical sampling, but $C$. sp. 24 and C. brenneri are found elsewhere in tropical latitudes $[35,36]$ (M. Rockman, pers. comm.). The species identified at the regional scale were a subset of those found within the more-intensely sampled Nouragues Natural Reserve locality. In only one instance can we point to clear substrate specificity: $C$. astrocarya n. sp. was isolated exclusively in the inflorescences of Astrocaryum palms, on which it frequently occurs. We also note that $C$. brenneri was found solely in sites impacted by humans, and never within the rainforest interior. This observation suggests that, like C. elegans, C. brenneri might experience dispersal benefits from human association. The lack of increasing species richness at larger spatial scales within French Guiana suggests that either (i) we failed to adequately sample the full diversity of microhabitats accessible to Caenorhabditis, (ii) the range of habitats and niche space for Caenorhabditis are relatively uniform across local and regional scales in this tropical region of South America, or (iii) dispersal of Caenorhabditis is high enough in this region to render all of it accessible for colonization.

The combination of field surveys and experiments showed that Caenorhabditis were rarely encountered in leaf litter, but comprised the predominant group of nematodes in rotting fruit $(2.7 \%$ of litter vs $20.6 \%$ of fruit samples had Caenorhabditis). Caenorhabditis also represented the dominant group that colonized fresh fruit baits, demonstrating that two of the most common species are capable of colonizing new resource patches rapidly ( 2.5-7.5\% baits/day; $C$. nouraguensis and $C$. tropicalis). These findings establish that Caenorhabditis colonizes new nutrient-rich substrates rapidly and more efficiently than do other kinds of nematodes. Nevertheless, given the observed colonization rates of fresh baits, many resource patches are likely to remain uncolonized by Caenorhabditis before complete fruit decay. As a result, resource exploitation by Caenorhabditis is likely to be limited by dispersal to fresh patches. Colonization of fresh resources occurred most readily when in proximity to pre-existing native fruit patches on the rainforest floor. This analysis thus provides quantitative support for the idea that Caenorhabditis occurs most frequently in high-nutrient rotting resources rich in active microbial growth, and that Caenorhabditis are not "soil nematodes" $[10,11,14]$. Future work should determine whether distinct types of fruit baits differentially attract Caenorhabditis and other nematode species and include more intensive analysis of invertebrate associations.

These observations about substrate incidence raise the question of the most likely source of fresh resource patch founding: vector-mediated colonization or a "seed bank" of dauer larvae that crawl through the leaf litter. If primarily by vectors, as known for species like temperate-zone C. japonica [37], are flying versus crawling invertebrates primarily involved? Direct experimental tests are required to confidently assess these possibilities. However, given the very low densities of Caenorhabditis found in leaf litter, we are skeptical that a "seed bank" of nematodes represents a dominant mode of colonization of new patches from crawling nematodes within the Nouragues Natural Reserve. Rainwater on vegetation and the ground might also provide a means of nematode dispersal. A remaining further question is whether Caenorhabditis might colonize resource patches prior to fruits and flowers falling to the rainforest floor, for example, from pollinators or frugivorous animals.

Despite successful colonization of fresh resource patches by Caenorhabditis, many substrate patches remained unoccupied in our bait sampling experiment. The apparently rapid decay of such rotting fruit substrates $(<1-2$ weeks) suggests that full exploitation of available resources by Caenorhabditis is likely to go unrealized due to imperfect colonization ability. Such dispersal limitation means that a single founder event of one or multiple individuals per patch is likely, which raises several interesting issues. First, the high turnover of individual resource patches, the hierarchical structuring of resource patches (individual fruits, individual source trees, landscape-scale clustering), and temporal dynamics in patch availability all make Caenorhabditis a tantalizing system for integrating metapopulation colonization-extinction processes with species co-existence [2, 10, 38, 39]. Second, priority effects for within-patch co-existence may be especially pronounced [40], also raising the potential for reproductive interference in accidental inter-species matings to interact with resource competition [41, 42]. Third, it remains unclear whether the succession of different species of Caenorhabditis (or other nematodes) might provide a means to facilitate co-existence and to limit direct competition, especially for larger resource patches or patches that decay more slowly. The dynamics of species turnover within resource patches remains to be determined. Fourth, when individual vector transport (e.g. insects) constrains the number of colonist nematodes that colonize a new resource patch, such founder 
events could provide a recurring selective pressure favoring selfing as a means of reproductive assurance in the face of high metapopulation turnover [43].

Caenorhabditis life history strategies span the generalist to specialist spectrum, which is predicted to associate with population demography and genetic variation [44]. Distinct reproductive modes, self-fertilizing hermaphroditism and obligate outbreeding, also occur in Caenorhabditis that exert profound effects on genome evolution $[44,45]$. In French Guiana, we find sympatric species spanning these traits and further find that the diverse Caenorhabditis community comprises a few abundant species and many numerically rare species. These attributes place these nematodes in a convenient position to test hypotheses about how organismal traits and population demography influence genetic variation and evolutionary change in the context of ecological community assembly $[10,44]$. We anticipate that future studies of this and other hotspots of Caenorhabditis diversity will provide important further contributions to understanding the inter-relation of ecological factors, organismal features, and genomic trends in evolution.

\section{Conclusions}

From nearly 3000 substrate samples in French Guiana, we isolated over 600 strains from nine species of Caenorhabditis nematodes, providing two new species in the largest biodiversity survey for these animals to date in habitat unperturbed by human activity. Experiments in the field demonstrate rapid colonization of nutrient-rich resources by Caenorhabditis, but imply that dispersal limitation will leave many resource patches unexploited by Caenorhabditis prior to their complete decay. These findings motivate deeper integration of field and lab experiments with multispecies Caenorhabditis communities to test ecological and evolutionary theory about metapopulation dynamics, priority effects, competition, species co-existence, and adaptation.

\section{Additional files}

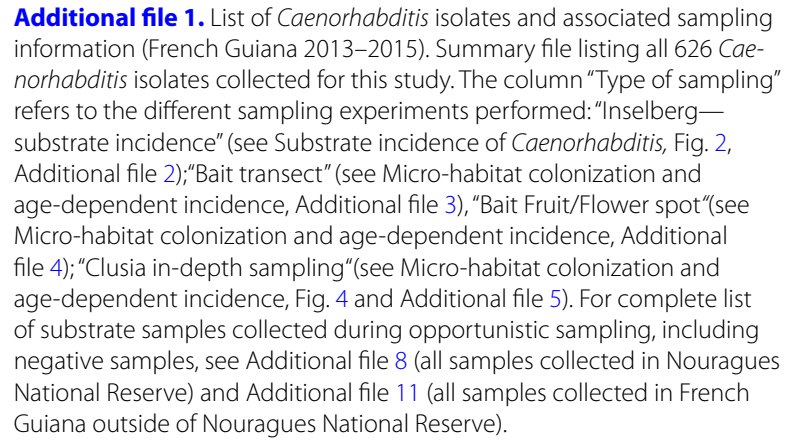

Additional file 1. List of Caenorhabditis isolates and associated sampling information (French Guiana 2013-2015). Summary file listing all 626 Caenorhabditis isolates collected for this study. The column "Type of sampling" refers to the different sampling experiments performed: "Inselbergsubstrate incidence" (see Substrate incidence of Caenorhabditis, Fig. 2, Additional file 2);"Bait transect" (see Micro-habitat colonization and age-dependent incidence, Additional file 3), "Bait Fruit/Flower spot"(see Micro-habitat colonization and age-dependent incidence, Additional file 4); "Clusia in-depth sampling"(see Micro-habitat colonization and age-dependent incidence, Fig. 4 and Additional file 5). For complete list of substrate samples collected during opportunistic sampling, including negative samples, see Additional file 8 (all samples collected in Nouragues National Reserve) and Additional file 11 (all samples collected in French Guiana outside of Nouragues National Reserve).

Additional file 2. Substrate incidence of Caenorhabditis (Inselberg site, Nouragues Natural Reserve). Raw and summary data that were used to estimate quantitative differences in the incidence of Caenorhabditis in decaying leaf litter versus rotting fruit. For labelling of intersections of sampled area, see Fig. 3.

Additional file 3. Micro-habitat colonization and age-dependent incidence: Caenorhabditis colonization of orange bait samples distributed at 72 spots along trail system. At each of the 72 spots (Parare, Nouragues Natural Reserve), three baits were distributed (i.e. subsamples, labelled a, b, c) approximately 1 meter apart from each other. See also Additional file 7.

Additional file 4. Micro-habitat colonization and age-dependent incidence: Caenorhabditis colonization of bait samples at spots with high fruit/flower density. We selected five localities near the Parare station and 18 localities near the Inselberg station (Nouragues Natural Reserve). At each locality, we collected five native fruit/flower samples as well as five (5-day old) bait samples within an area of approximately $5 \mathrm{~m}^{2}$. See also Additional file 4

Additional file 5. Micro-habitat colonization and age-dependent incidence: Caenorhabditis colonization as a function of substrate age (Clusia flowers). To estimate Caenorhabditis colonization as a function of substrate age, we haphazardly collected 70 flower samples of each of three clearly distinct, progressive stages of decay $(\mathrm{N}=210)$ at a single locality. Labelling of samples is arbitrary. See also Fig. 4.

Additional file 6. Micro-habitat colonization and age-dependent incidence: Population census and developmental stages of Caenorhabditis colonizing Clusia flowers. In addition to the Clusia colonization assessment (Fig. 4, Additional file 4), we collected 21 Clusia flower samples containing Caenorhabditis $(\mathrm{N}=21)$ and immediately placed them on NGM plates seeded with E. coli to determine number and stages of individuals $5-7 \mathrm{~h}$ later.

Additional file 7. Micro-habitat colonization and age-dependent incidence: Map of Caenorhabditis species recovery on baits $(\mathrm{N}=216)$ distributed at 72 spots along trail system (Parare, Nouragues Natural Reserve). At each of the 72 spots (Parare, Nouragues Natural Reserve), three baits were distributed (i.e. subsamples, labelled a, b, c) approximately 1 meter apart from each other. Each sampling spot is indicated by a single dot, indicating species occurring in the three samples. At one site (\#60), different samples contained C. briggsae and C. tropicalis. At another site (\#59), a single subsample (59b) contained both C. nouraguensis and C. tropicalis. See Additional file 3 for detailed information.

Additional file 8. List of substrate samples and Caenorhabditis isolates collected in the Nouragues National Reserve (2013-2015).

Additional file 9. Map of opportunistic samples and Caenorhabditis species distribution at the Inselberg site (Nouragues Natural Reserve) $(\mathrm{N}=278)$.

Additional file 10. Map of opportunistic samples and Caenorhabditis species distribution at the Parare site (Nouraques Natural Reserve) $(\mathrm{N}=313)$.

Additional file 11. List of substrate samples and Caenorhabditis isolates collected in the coastal regions of French Guiana and in the area of Saul in central French Guiana $(2013,2015)(N=457)$.

Additional file 12. Crosses of novel species (C. dolens, C. astrocarya) with known Caenorhabditis species [3]. Reciprocal crosses between different species and strains were performed as previously described [3], allowing for mating between 5-10 males and 5-10 females on a $55 \mathrm{~mm}$ diameter NGM plate. (A) Results of reciprocal crosses between the novel species $C$. dolens and presumptively close known relatives $C$. angaria, C. castelli, $C$ sp. 8, and C. astrocarya (B) Results of crosses between the novel species $C$. astrocarya and close known relatives, C. guadeloupensis and C. dolens.

Additional file 13. DIC microscopy images of C. dolens sp. n. (strain NIC394). (A) Ventral view of the adult male tail showing multiple ventral genital papillae. (B) Lateral view of adult male tail, focused on spicule. (C) Lateral view of buccal region (stoma) of adult female. (D) Ventral view of 
external vulval opening and cuticle of an adult female. (E) Lateral view of mid-body region of a young adult female showing vulva, uterus and germline (a maturing oocyte and spermatheca filled with sperm are visible).

Additional file 14. DIC microscopy images of C. astrocarya sp. n. (strain NIC1040). (A) Ventral view of the adult male tail showing multiple ventral and dorsal genital papillae. (B) Lateral view of adult male tail, focused on spicule, with several dorsal genital papillae visible. (C) Lateral view of pharynx region of an adult female. (D) Ventral view of mid-body region of any adult female showing vulva opening (to the right, two young embryos, and to the left, the spermatheca, are visible). (E) Lateral view of buccal region (stoma) of adult female.

Additional file 15. Images of Astrocaryum palms (likely Astrocaryum paramaca) and inflorescence, representing the micro-habitat of the novel species, C. astrocarya (Nouragues Natural Reserve).

\section{Authors' contributions}

CF and RS performed data collection and analysis in the lab. NC and RJ performed field experiments and collected data. AC and CB designed the experiments, collected and analyzed data, and wrote the manuscript. All authors read and approved the final manuscript.

\section{Acknowledgements}

We thank the CNRS in French Guiana and its staff, in particular, Patrick Châtelet, Jérôme Chave, Philippe Gaucher, Annaig Le Guen and Laetitia Proux. We further acknowledge support and advice provided by the Parc Amazonien de Guyane (Raphaëlle Rinaldo) and the Collectivité Territoriale de Guyane (Frédéric Blanchard). We thank two anonymous reviewers and Marie-Anne Félix for helpful comments on previous versions of the manuscript, Marine Guntz for help with sampling and Anne Vielle, Clotilde Gimond, Paul Vigne and Emilie Demoinet for help with freezing numerous Caenorhabditis isolates.

\section{Availability of data and materials}

Partial ITS2 sequences of the two new species (C. astrocarya and C. dolens) are deposited in GenBank (accessions MF940414-MF940415). This published work and the nomenclatural acts it contains have been registered in ZooBank, the online registration system for the ICZN. The ZooBank LSIDs (Life Science Identifiers) can be resolved and the associated information viewed through any standard web browser by appending the LSID to the prefix "http://zoobank. org/". The LSID for this publication is urn:Isid:zoobank.org:pub:06DBDCA0DD60-43BA-9A99-14F0748D4FE2. The ZooBank LSID for Caenorhabditis dolens is urn:Isid:zoobank.org:act:4D39DD5D-2120-42E5-8521-0F35D8710592 and the the LSID for Caenorhabditis astrocarya is urn:Isid:zoobank.org:act:EBE4FF82F906-4237-9290-C84431F17D7D. The type culture specimens are deposited at the Caenorhabditis Genetics Center (http://www.cbs.umn.edu/research/ resources/cgc). Live strain isolates are available from the authors. Complete collection data are provided as supplementary material in Additional files 1, 8 and 11).

\section{Competing interests}

The authors declare that they have no competing interests.

\section{Ethics approval and consent to participate}

Collection of samples in Saül and surroundings were conducted with authorization of the Parc Amazonien de Guyane (APA-973-5).

\section{Consent to publish}

Not applicable.

\section{Funding}

This work was made possible through grants provided by the LABEX CEBA (Agence Nationale de la Recherche), the France-Canada Research Foundation and the Nouragues Travel Grant Program (CNRS).

\section{Publisher's Note}

Springer Nature remains neutral with regard to jurisdictional claims in published maps and institutional affiliations.
Received: 29 May 2017 Accepted: 6 December 2017

Published online: 19 December 2017

\section{References}

1. Shurin JB, Amarasekare P, Chase JM, Holt RD, Hoopes MF, Leibold MA. Alternative stable states and regional community structure. J Theor Biol. 2004;227(3):359-68.

2. Case TJ. Invasion resistance, species build-up and community collapse in metapopulation models with interspecies competition. Biol J Linn Soc. 1991;42(1-2):239-66.

3. Yu DW, Wilson HB. The competition-colonization trade-off is dead; long live the competition-colonization trade-off. Am Nat. 2001;158(1):49-63.

4. Felix M-A, Jovelin R, Ferrari C, Han S, Cho YR, Andersen EC, Cutter AD, Braendle C. Species richness, distribution and genetic diversity of Caenorhabditis nematodes in a remote tropical rainforest. BMC Evol Biol. 2013;13(1):10.

5. Felix MA, Braendle C, Cutter AD. A streamlined system for species diagnosis in Caenorhabditis (Nematoda: Rhabditidae) with name designations for 15 distinct biological species. PLoS ONE. 2014;9:e94723.

6. Kiontke K, Félix M-A, Ailion M, Rockman MV, Braendle C, Pénigault J-B, Fitch DH. A phylogeny and molecular barcodes for Caenorhabditis, with numerous new species from rotting fruits. BMC Evol Biol. 2011;11:339.

7. Cutter AD, Dey A, Murray RL. Evolution of the Caenorhabditis elegans genome. Mol Biol Evol. 2009;26(6):1199-234.

8. Cutter AD. Divergence times in Caenorhabditis and Drosophila inferred from direct estimates of the neutral mutation rate. Mol Biol Evol. 2008;25(4):778-86.

9. Kaletta T, Hengartner MO. Finding function in novel targets: C. elegans as a model organism. Nature Rev Drug Discov. 2006;5(5):387-99.

10. Cutter AD. Caenorhabditis evolution in the wild. BioEssays. 2015;37(9):983-95.

11. Frézal L, Félix M-A. C. elegans outside the Petri dish. eLife. 2015;4:e05849.

12. Barrière A, Félix M-A. Temporal dynamics and linkage disequilibrium in natural C. elegans populations. Genetics. 2007;176:999-1011.

13. Schulenburg H, Felix MA. The natural biotic environment of Caenorhabditis elegans. Genetics. 2017;206(1):55-86.

14. Felix MA, Braendle C. The natural history of Caenorhabditis elegans. Curr Biol. 2010;20(22):R965-9.

15. Barrière $A$, Félix M-A. High local genetic diversity and low outcrossing rate in Caenorhabditis elegans natural populations. Curr Biol. 2005;15:1176-84.

16. Félix MA, Duveau F. Population dynamics and habitat sharing of natural populations of Caenorhabditis elegans and C. briggsae. BMC Biol. 2012;10(1):59.

17. Petersen C, Dirksen P, Prahl S, Strathmann E, Schulenburg H. The prevalence of Caenorhabditis elegans across 1.5 years in selected North German locations: the importance of substrate type, abiotic parameters, and Caenorhabditis competitors. BMC Ecol. 2014;14(1):4.

18. Samuel BS, Rowedder H, Braendle C, Félix MA, Ruvkun G. Caenorhabditis elegans responses to bacteria from its natural habitats. Proc Nat Acad Sci. 2016;113(27):E3941-9.

19. Braendle C, Teotonio H. Workshop report: Caenorhabditis nematodes as model organisms to study trait variation and its evolution. Worm. 2015;4:e1021109.

20. Thomas CG, Wang W, Jovelin R, Ghosh R, Lomasko T, Trinh Q, Kruglyak L, Stein LD, Cutter AD. Full-genome evolutionary histories of selfing, splitting and selection in Caenorhabditis. Genome Res. 2015;25:667-78.

21. Andersen EC, Gerke JP, Shapiro JA, Crissman JR, Ghosh R, Bloom JS, Felix MA, Kruglyak L. Chromosome-scale selective sweeps shape Caenorhabditis elegans genomic diversity. Nat Genet. 2012;44:285-90.

22. Cook DE, Zdraljevic S, Tanny RE, Seo B, Riccardi DD, Noble LM, Rockman MV, Alkema MJ, Braendle C, Kammenga JE, et al. The genetic basis of natural variation in Caenorhabditis elegans telomere length. Genetics. 2016;204(1):371-83.

23. Felix MA, Ashe A, Piffaretti J, Wu G, Nuez I, Belicard T, Jiang Y, Zhao G, Franz CJ, Goldstein LD, et al. Natural and experimental infection of Caenorhabditis nematodes by novel viruses related to nodaviruses. PLoS Biol. 2011;9(1):e1000586. 
24. Haber M, Schüngel M, Putz A, Müller S, Hasert B, Schulenburg H. Evolutionary history of Caenorhabditis elegans inferred from microsatellites: evidence for spatial and temporal genetic differentiation and the occurrence of outbreeding. Mol Biol Evol. 2005;22:160-73.

25. Petersen C, Saebelfeld M, Barbosa C, Pees B, Hermann RJ, Schalkowski R, Strathmann EA, Dirksen $\mathrm{P}$, Schulenburg $\mathrm{H}$. Ten years of life in compost: temporal and spatial variation of North German Caenorhabditis elegans populations. Ecol Evol. 2015;5(16):3250-63.

26. Petersen C, Hermann RJ, Barg M-C, Schalkowski R, Dirksen P, Barbosa C, Schulenburg H. Travelling at a slug's pace: possible invertebrate vectors of Caenorhabditis nematodes. BMC Ecol. 2015;15:1-13.

27. Gimond C, Jovelin R, Han S, Ferrari C, Cutter AD, Braendle C. Outbreeding depression with low genetic variation in selfing Caenorhabditis nematodes. Evolution. 2013;67(11):3087-101.

28. Poullet N, Braendle C. Sampling and isolation of C. elegans from the natural habitat. Methods Mol Biol. 2015;1327:221-9.

29. Barrière A, Félix MA. Isolation of C. elegans and related nematodes. WormBook. 2006:17:1-9.

30. Stiernagle T. Maintenance of C. elegans. In: WormBook. Edited by the C. elegans Research Community: WormBook; 2005.

31. Barrière A, Félix M-A: Isolation of C. elegans and related nematodes. In: Wormbook. Edited by Community TCeR; 2006.

32. Colwell RK, Elsensohn JE. EstimateS turns 20: statistical estimation of species richness and shared species from samples, with non-parametric extrapolation. Ecography. 2014;37(6):609-13.

33. Gonçalves-alvim SD. Resin-collecting bees (Apidae) on Clusia palmicida (Clusiaceae) in a riparian forest in Brazil. J Trop Ecol. 2001;17(1):149-53.

34. Magurran AE. Measuring biological diversity. Oxford: Blackwell Science; 2004

35. Dey A, Chan CK-W, Thomas CG, Cutter AD. Nucleotide hyperdiversity defines populations of Caenorhabditis brenneri. Proce Nat Acad Sci USA. 2013;110:11056-60.
36. Sudhaus W, Kiontke K. Comparison of the cryptic nematode species Caenorhabditis brenneri sp. n. and C. remanei (Nematoda: Rhabditidae) with the stem species pattern of the Caenorhabditis Elegans group. Zootaxa. 2007; 1456:45-62.

37. Yoshiga T, Ishikawa Y, Tanaka R, Hironaka M, Okumura E. Species-specific and female host-biased ectophoresy in the roundworm Caenorhabditis japonica. Naturwissenschaften. 2013;100(2):205-8.

38. Fournier B, Mouquet N, Leibold MA, Gravel D. An integrative framework of coexistence mechanisms in competitive metacommunities. Ecography. 2017;40(5):630-41.

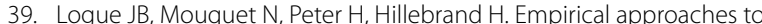
metacommunities: a review and comparison with theory. Trends Ecol Evol. 2011;26(9):482-91.

40. De Meester L, Vanoverbeke J, Kilsdonk LJ, Urban MC. Evolving perspectives on monopolization and priority effects. Trends Ecol Evol. 2016:31(2):136-46.

41. Ting JJ, Woodruff GC, Leung G, Shin N-R, Cutter AD, Haag ES. Intense sperm-mediated sexual conflict promotes gametic isolation in Caenorhabditis nematodes. PLoS Biol. 2014;12:e1001915.

42. Kishi S, Nakazawa T. Analysis of species coexistence co-mediated by resource competition and reproductive interference. Popul Ecol. 2013;55(2):305-13.

43. Pannell JR, Barrett SCH. Baker's Law revisited: reproductive assurance in a metapopulation. Evolution. 1998;52(3):657-68.

44. Li S, Jovelin R, Yoshiga T, Tanaka R, Cutter AD. Specialist versus generalist life histories and nucleotide diversity in Caenorhabditis nematodes. Proc R Soc LonD B Biol Sci. 2014;281(1777):20132858.

45. Fierst $J$, Willis JH, Thomas CG, Wang W, Reynolds RM, Ahearne T, Cutter AD, Phillips PC. Reproductive mode and the evolution of genomic size and structure in Caenorhabditis nematodes. PLoS Genet. 2015;11:e1005323.

\section{Submit your next manuscript to BioMed Central and we will help you at every step:}

- We accept pre-submission inquiries

- Our selector tool helps you to find the most relevant journal

- We provide round the clock customer support

- Convenient online submission

- Thorough peer review

- Inclusion in PubMed and all major indexing services

- Maximum visibility for your research

Submit your manuscript at www.biomedcentral.com/submit
O Biomed Central 